\title{
ASYMPTOTIC BEHAVIOR OF EIGENVALUES OF RECIPROCAL POWER LCM MATRICES
}

\author{
SHAOFANG HONG \\ Mathematical College, Sichuan University, Chengdu 610064, P.R. China \\ e-mail:s-f.hong@tom.com; hongsf02@yahoo.com
}

and K. S. ENOCH LEE

Department of Mathematics, Auburn University Montgomery, Montgomery, AL 36124-4023, USA

e-mail: elee4@mail.aum.edu

(Received 12 April, 2007; revised 15 August, 2007; accepted 25 August, 2007)

\begin{abstract}
Let $\left\{x_{i}\right\}_{i=1}^{\infty}$ be an arbitrary strictly increasing infinite sequence of positive integers. For an integer $n \geq 1$, let $S_{n}=\left\{x_{1}, \ldots, x_{n}\right\}$. Let $r>0$ be a real number and $q \geq 1$ a given integer. Let $\lambda_{n}^{(1)} \leq \ldots \leq \lambda_{n}^{(n)}$ be the eigenvalues of the reciprocal power LCM matrix $\left(\frac{1}{\left[x_{i}, x_{j}\right]^{]}}\right)$having the reciprocal power $\frac{1}{\left[x_{i}, x_{j}\right]^{r}}$ of the least common multiple of $x_{i}$ and $x_{j}$ as its $i$,j-entry. We show that the sequence $\left\{\lambda_{n}^{(q)}\right\}_{n=q}^{\infty}$ converges and $\lim _{n \rightarrow \infty} \lambda_{n}^{(q)}=0$. We show that the sequence $\left\{\lambda_{n}^{(n-q+1)}\right\}_{n=q}^{\infty}$ converges if $s_{r}:=\sum_{i=1}^{\infty} \frac{1}{x_{i}^{r}}<\infty$ and $\lim _{n \rightarrow \infty} \lambda_{n}^{(n-q+1)} \leq s_{r}$. We show also that if $r>1$, then the sequence $\left\{\lambda_{l n}^{(t n-q+1)}\right\}_{n=1}^{\infty}$ converges and $\lim _{n \rightarrow \infty} \lambda_{l n}^{(t n-q+1)}=0$, where $t$ and $l$ are given positive integers such that $t \leq l-1$.
\end{abstract}

2000 Mathematics Subject Classification. Primary 11C20, 11A05, 15A36.

1. Introduction. Let $1 \leq x_{1}<\ldots<x_{n}<\ldots$ be a given arbitrary strictly increasing infinite sequence of positive integers. For any integer $n \geq 1$, let

$$
S_{n}=\left\{x_{1}, \ldots, x_{n}\right\} .
$$

Now let $r>0$ be a real number. The $n \times n$ matrix having the power $\left(x_{i}, x_{j}\right)^{r}$ of the greatest common divisor of $x_{i}$ and $x_{j}$ as its $i, j$-entry is called the power greatest common divisor $(G C D)$ matrix defined on $S_{n}$, denoted by $\left(\left(x_{i}, x_{j}\right)^{r}\right)$, or abbreviated by $\left(\left(S_{n}\right)^{r}\right)$. The matrix having the power $\left[x_{i}, x_{j}\right]^{r}$ of the least common multiple of $x_{i}$ and $x_{j}$ as its $i, j$ entry is called the power least common multiple (LCM) matrix, denoted by $\left(\left[x_{i}, x_{j}\right]^{r}\right)$, or abbreviated by $\left[\left(S_{n}\right)^{r}\right]$. If we let $r=1$, then the power GCD matrix and the power LCM matrix are said to be the GCD matrix defined on $S_{n}$ and the LCM matrix defined on $S_{n}$, respectively, and denoted by $\left(S_{n}\right)$ and $\left[S_{n}\right]$, respectively. Smith was the first mathematician who studied the power GCD matrices and power LCM matrices (see [9, 33, 35]). Later on, many generalizations of Smith's determinant have been published, see $[1,2,4-8,11,12,14-26,29-32,34,36,37]$ and the references listed there. In 1989, Beslin and Ligh [4] initiated the study of the GCD matrix $\left(S_{n}\right)$ on any set $S_{n}$ in the direction of structure, determinant and inverse. In particular, they proved

The corresponding author is S. Hong who was supported by Program for New Century Excellent Talents in University Grant \# NCET-06-0785 and by SRF for ROCS, SEM. 
that the GCD matrix $\left(S_{n}\right)$ on any set $S_{n}$ of $n$ distinct positive integers is positive definite. However, the LCM matrix $\left[S_{n}\right]$ on any set $S_{n}$ is not positive definite in general. It may not even be nonsingular. In fact, Hong [16] showed that the LCM matrix $\left[S_{n}\right]$ defined on any gcd-closed set $S_{n}$ (i.e. $\left(x_{i}, x_{j}\right) \in S_{n}$ for all $\left.1 \leq i, j \leq n\right)$ is nonsingular if $n \leq 7$, and for every integer $n \geq 8$, there exist gcd-closed sets $S_{n}$ such that the LCM matrix $\left[S_{n}\right]$ on $S_{n}$ is singular (see [16, 21, 22]). Notice that Haukkanen et al. [12] also found a gcd-closed set $S_{n}$ such that the LCM matrix [ $S_{n}$ ] on $S_{n}$ is singular when $n=9$. Recently Hong [23] proved that there are gcd-closed sets $S_{n}$ and lcm-closed sets $S_{n}$ (i.e. $\left[x_{i}, x_{j}\right] \in S_{n}$ for all $1 \leq i, j \leq n)$ such that the reciprocal GCD matrix $\left(\frac{1}{\left(S_{n}\right)}\right)$ on $S_{n}$ is singular.

For the following form of a power GCD matrix

$$
N_{n}:=\left(\frac{(i, j)^{2 r}}{i^{r} \cdot j^{r}}\right)_{1 \leq i, j \leq n}=\left(\frac{(i, j)^{r}}{[i, j]^{r}}\right)_{1 \leq i, j \leq n},
$$

Wintner [36] proved in 1944 that $\lim _{\sup _{n \rightarrow \infty}} \Lambda_{n}(r)<\infty$ if and only if $r>1$, where $\Lambda_{n}(r)$ denotes the largest eigenvalue of the matrix $N_{n}$. Let $\lambda_{n}(r)$ denote the smallest eigenvalue of the matrix $N_{n}$. Lindqvist and Seip [31] in 1998 used the work of [13] about Riesz bases to investigate the asymptotic behavior of $\lambda_{n}(r)$ and $\Lambda_{n}(r)$ as $n \rightarrow \infty$. In particular, they got a sharp bound for $\lambda_{n}(r)$ and $\Lambda_{n}(r)$. In 1993, Bourque and Ligh [5] extended Beslin and Ligh's result [4] by showing that for any $r>0$, the power GCD matrix $\left(\left(S_{n}\right)^{r}\right)$ on $S_{n}$ is positive definite. From this, one can only conclude that its eigenvalues are positive. Hong and Loewy [24, 25] investigated the eigen-structure of the power GCD matrices and made some significant progress in this topic. In fact, it was proved in [24] that if $0<r \leq 1$ and $q \geq 1$ is any fixed integer, then the $q$-th smallest eigenvalue of the $n \times n$ power GCD matrix $\left((i, j)^{r}\right)$ approaches zero as $n$ tends to infinity. We also note that Cao [8], Hong [20], Hong-Shum-Sun [26] and Li [30] investigated the nonsingularity of power LCM matrices while Hong [18, 19], Haukkanen-Korkee [11] and Zhao-Hong-Liao-Shum [37] studied the divisibility of power LCM matrices by power GCD matrices.

In the present paper, our main aim is to consider the asymptotic behavior of eigenvalues of reciprocal power LCM matrices. Let $r>0$ be a real number and $q \geq 1$ a given integer. Let $\lambda_{n}^{(1)} \leq \ldots \leq \lambda_{n}^{(n)}$ be the eigenvalues of the reciprocal power LCM matrix $\left(\frac{1}{\left[x_{i}, x_{j}\right]^{r}}\right)$ having the reciprocal power $\frac{1}{\left[x_{i}, x_{j}\right]^{r}}$ of the least common multiple of $x_{i}$ and $x_{j}$ as its $i, j$-entry. We show that the sequence $\left\{\lambda_{n}^{(q)}\right\}_{n=q}^{\infty}$ converges and $\lim _{n \rightarrow \infty} \lambda_{n}^{(q)}=0$. We show also that the sequence $\left\{\lambda_{n}^{(n-q+1)}\right\}_{n=q}^{\infty}$ converges if $\sum_{i=1}^{\infty} \frac{1}{x_{i}^{r}}<\infty$ and $\lim _{n \rightarrow \infty} \lambda_{n}^{(n-q+1)} \leq \sum_{i=1}^{\infty} \frac{1}{x_{i}^{r}}$. Finally we investigate asymptotic behavior of the mediate eigenvalues of reciprocal power LCM matrices. Actually we show that if $r>1$, then the sequence $\left\{\lambda_{l n}^{(t n-q+1)}\right\}_{n=1}^{\infty}$ converges and $\lim _{n \rightarrow \infty} \lambda_{l n}^{(t n-q+1)}=0$, where $t$ and $l$ are given positive integers such that $t \leq l-1$.

2. On the smallest eigenvalue of reciprocal power LCM matrices. We first show that the reciprocal power LCM matrix defined on any set of positive integers is positive definite. We also give bounds for its determinant. Denote by $\operatorname{gcd}(A)$ the greatest common divisor of the elements of a finite set $A$ of positive integers.

THEOREM 2.1. Let $r>0$ be a real number and $S_{n}=\left\{x_{1}, \ldots, x_{n}\right\}$ be a set of $n$ distinct positive integers. Then each of the following is true: 
(i) $\prod_{k=1}^{n} \frac{1}{x_{k}^{2 r}} \sum_{I \subseteq\left\{x \mid x \in S_{n}, x<x_{k}\right\}}(-1)^{|I|}\left(\operatorname{gcd}\left(\left\{x_{k}\right\} \cup I\right)\right)^{r} \leq \operatorname{det}\left(\frac{1}{\left[x_{i}, x_{j}\right]^{r}}\right) \leq \prod_{k=1}^{n} \frac{1}{x_{k}^{r}}$. Furthermore, the equality on the left-hand side inequality holds if and only if $S_{n}$ is gcd closed.

(ii) The $n \times n$ matrix $\left(\frac{1}{\left[x_{i}, x_{j}\right]^{r}}\right)$ is positive definite.

Proof. Since $x_{i} x_{j}=\left[x_{i}, x_{j}\right]\left(x_{i}, x_{j}\right)$ for all $1 \leq i, j \leq n$, we get

$$
\left(\frac{1}{\left[x_{i}, x_{j}\right]^{r}}\right)=D \cdot\left(\left(x_{i}, x_{j}\right)^{r}\right) \cdot D
$$

where $D=\operatorname{diag}\left(\frac{1}{x_{1}^{r}}, \ldots, \frac{1}{x_{n}^{r}}\right)$. Then we derive that

$$
\operatorname{det}\left(\frac{1}{\left[x_{i}, x_{j}\right]^{r}}\right)=\operatorname{det}\left(\left(x_{i}, x_{j}\right)^{r}\right) \cdot \prod_{k=1}^{n} \frac{1}{x_{k}^{2 r}} .
$$

By Theorem 1 of [15] we have $\operatorname{det}\left(\left(x_{i}, x_{j}\right)^{r}\right) \geq \prod_{k=1}^{n} \alpha_{k, r}$, where

$$
\alpha_{k, r}=\sum_{\substack{d \mid x_{k} \\ d x_{t}, x_{t}<x_{k}}} J_{r}(d)
$$

and $J_{r}$ denotes the generalized Jordan function, i.e. $J_{r}=\xi_{r} * \mu$ with $\xi_{r}(m)=m^{r}$ for any positive integer $m$. But Claim 2.2 of [21] applied to $f=\xi_{r}$ gives us that (note that Claim 2.2 of [21] is stated under the condition that $S_{n}$ is gcd closed but clearly this is not necessary)

$$
\alpha_{k, r}=\sum_{I \subseteq\left\{x \mid x \in S_{n}, x<x_{k}\right\}}(-1)^{|I|}\left(\operatorname{gcd}\left(\left\{x_{k}\right\} \cup I\right)\right)^{r} .
$$

Thus the inequality on the left-hand side of part (i) follows immediately. Furthermore, by Theorem 1 of [15] we know that the equality in the above inequality holds if and only if $S_{n}$ is ged closed. Thus the second assertion of part (i) follows immediately.

For any $1 \leq l \leq n$, the inequality on the left-hand side of (i) implies that the determinant of any principal submatrix of order $l$ of $\left(\frac{1}{\left[x_{i}, x_{j}\right]^{]}}\right)$is positive. This concludes part (ii). From (ii) the inequality on the right-hand side of (i) follows immediately. Hence the proof of Theorem 2.1 is complete.

REMARK. In Theorem 2.1, the condition $r>0$ is necessary. In fact, letting $r<0$ gives us a power LCM matrix $\left(\left[x_{i}, x_{j}\right]^{-r}\right)$. As mentioned in the introduction section, this matrix is not necessarily positive definite. It may even be singular, see $[\mathbf{1 6}, \mathbf{2 1}, \mathbf{2 2}]$ for the examples when $r=-1$. On the other hand, it follows from part (ii) of Theorem 2.1 that Conjectures 4.1 and 4.5 of Hong [21] are true when $\varepsilon<0$ (see [21]). Note that Li [30] made some progress towards Conjectures 4.1 and 4.5 of Hong [21] when $\varepsilon>0$. Given $d, x \in S_{n}$ with $d<x$, we say that $d$ is a greatest-type divisor of $x$ in $S_{n}$ if $d \mid x$ and there is no other $y \in S_{n}$ such that $d \mid y$ and $y \mid x$ (see [16, 21-23]). We also remark that if $S_{n}$ is gcd closed, then by Theorem 1.2 of [21] we have the following further reduced formula:

$$
\alpha_{k, r}=\sum_{I \subseteq G_{S_{n}}\left(x_{k}\right)}(-1)^{|I|}\left(\operatorname{gcd}\left(\left\{x_{k}\right\} \cup I\right)\right)^{r},
$$

where $G_{S_{n}}\left(x_{k}\right)$ means the set of all greatest-type divisors of $x_{k}$ in the set $S_{n}$. 
Consequently, we investigate the asymptotic behavior of the smallest eigenvalue of the reciprocal power LCM matrices. We have the following result.

THEOREM 2.2. Let $r>0$ be a real number, $q \geq 1$ a given arbitrary integer and $\left\{x_{i}\right\}_{i=1}^{\infty}$ an arbitrary given strictly increasing infinite sequence of positive integers. Let $\lambda_{n}^{(1)} \leq \ldots \leq \lambda_{n}^{(n)}$ be the eigenvalues of the $n \times n$ reciprocal power LCM matrix $\left(\frac{1}{\left[x_{i}, x_{j}\right]^{r}}\right)$ defined on the set $S_{n}:=\left\{x_{1}, \ldots, x_{n}\right\}$. Then each of the following is true:

(i) We have

$$
0<\lambda_{n}^{(1)} \leq \frac{1}{n}\left(\frac{1}{x_{1}^{r}}+\cdots+\frac{1}{x_{n}^{r}}\right)
$$

(ii) The sequence $\left\{\lambda_{n}^{(q)}\right\}_{n=q}^{\infty}$ converges. Further, we have $\lim _{n \rightarrow \infty} \lambda_{n}^{(q)}=0$.

Proof. Let $1 \leq q \leq n$ be a fixed integer and $r>0$. Then it follows from part (ii) of Theorem 2.1 that the reciprocal power LCM matrix $\left(\frac{1}{\left[x_{i}, x_{j}\right]^{r}}\right)$ is positive definite and so we have

$$
\lambda_{n}^{(q)}>0
$$

On the other hand, by Cauchy's interlacing inequalities (see [27] and a new proof of it, see [28]) we have

$$
\lambda_{n+1}^{(q)} \leq \lambda_{n}^{(q)}
$$

Thus the sequence $\left\{\lambda_{n}^{(q)}\right\}_{n=q}^{\infty}$ is a non-increasing infinite sequence of positive real numbers and so it is convergent. In what follows we use induction on $q$ to show the second statement. First we prove the case $q=1$, i.e. $\lim _{n \rightarrow \infty} \lambda_{n}^{(1)}=0$. Since $\lambda_{n}^{(1)} \leq \cdots \leq \lambda_{n}^{(n)}$ and

$$
\lambda_{n}^{(1)}+\cdots+\lambda_{n}^{(n)}=\frac{1}{\left[x_{1}, x_{1}\right]^{r}}+\cdots+\frac{1}{\left[x_{n}, x_{n}\right]^{r}}=\frac{1}{x_{1}^{r}}+\cdots+\frac{1}{x_{n}^{r}},
$$

we get

$$
\lambda_{n}^{(1)} \leq \frac{1}{n}\left(\frac{1}{x_{1}^{r}}+\cdots+\frac{1}{x_{n}^{r}}\right) .
$$

Hence part (i) is proved. Since $r>0$ and $x_{i} \geq i$ for all integer $i \geq 1$, we then deduce that

$$
\lambda_{n}^{(1)} \leq \frac{1}{n}\left(1+\frac{1}{2^{r}}+\cdots+\frac{1}{n^{r}}\right) .
$$

Let first $r \geq 1$. Then by (3) we have

$$
\lambda_{n}^{(1)} \leq \frac{1}{n}\left(1+\frac{1}{2}+\cdots+\frac{1}{n}\right) .
$$

But it is well known (see [10] or part (a) of Theorem 3.2 of [3]) that

$$
1+\frac{1}{2}+\cdots+\frac{1}{n}=\log n+\gamma+O\left(\frac{1}{n}\right)
$$


where $\gamma$ is Euler's constant. So there is a constant $C>0$ such that

$$
1+\frac{1}{2}+\cdots+\frac{1}{n} \leq \log n+\gamma+\frac{C}{n} .
$$

Therefore

$$
\lambda_{n}^{(1)} \leq \frac{\log n}{n}+\frac{\gamma}{n}+\frac{C}{n^{2}}
$$

Then taking the limit in (1) (let $q=1$ ) and (4) gives $\lim _{n \rightarrow \infty} \lambda_{n}^{(1)}=0$ as required.

Now let $0<r<1$. By part (b) of Theorem 3.2 of [3] we have

$$
1+\frac{1}{2^{r}}+\cdots+\frac{1}{n^{r}}=\frac{n^{1-r}}{1-r}+\zeta(r)+O\left(\frac{1}{n^{r}}\right),
$$

where $\zeta(r)$ is the Riemann zeta function. Then there is a constant $C^{\prime}>0$ such that

$$
\lambda_{n}^{(1)} \leq \frac{1}{n}\left(\frac{n^{1-r}}{1-r}+\zeta(r)+\frac{C^{\prime}}{n^{r}}\right)=\frac{1}{n^{r}(1-r)}+\frac{\zeta(r)}{n}+\frac{C^{\prime}}{n^{1+r}} .
$$

Thus the desired result $\lim _{n \rightarrow \infty} \lambda_{n}^{(1)}=0$ follows immediately by taking the limit in (1) (let $q=1$ ) and (5).

Assume that $\lim _{n \rightarrow \infty} \lambda_{n}^{(k)}=0$ for $1 \leq k<q$. Now we consider the case $k=q$. Since $\lambda_{n}^{(q)} \leq \ldots \leq \lambda_{n}^{(n)}$, then from (2) we deduce that

$$
\lambda_{n}^{(1)}+\cdots+\lambda_{n}^{(q-1)}+(n-q+1) \lambda_{n}^{(q)} \leq 1+\frac{1}{2^{r}}+\cdots+\frac{1}{n^{r}} .
$$

So we have

$$
\frac{1}{n-q+1}\left(\lambda_{n}^{(1)}+\cdots+\lambda_{n}^{(q-1)}\right)+\lambda_{n}^{(q)} \leq \frac{1}{n-q+1}\left(1+\frac{1}{2^{r}}+\cdots+\frac{1}{n^{r}}\right) .
$$

In the same way as above, we can prove that the right-hand side of (6) approaches zero when $n$ goes to infinity since $r>0$. Note that the left-hand side of (6) is positive and $\lim _{n \rightarrow \infty} \lambda_{n}^{(k)}=0$ for $1 \leq k \leq q-1$. Therefore taking the limit in (6) gives us $\lim _{n \rightarrow \infty} \lambda_{n}^{(q)}=0$. This completes the proof of part (ii) of Theorem 2.2.

REMARK. Associated to an arbitrary given strictly increasing infinite sequence of positive integers, we can form an infinite sequence of power GCD matrices and an infinite sequence of reciprocal power LCM matrices. Hong and Loewy [24] proved that the sequence of the smallest eigenvalues of such power GCD matrices converges and the limit is nonnegative when $n$ approaches infinity. But the limit may be positive (see Theorem 2.5 of [24]). However, by Theorem 2.2 (ii) we know that the limit of the smallest eigenvalue of such reciprocal power LCM matrices is zero as $n$ goes to infinity.

The above proofs are number-theoretic. In what follows we give a matrix analysis proof to the second assertion of part (ii) of Theorem 2.2. Indeed, we prove the following result which gives an upper bound of $\lambda_{n}^{(k)}$ for $1 \leq k \leq n$ with a different flavor. Note that the following result is sharper than that of Theorem 2.2 for the case $k=1$. Furthermore, we provide a lower bound for $\lambda_{n}^{(n)}$. 
THEOREM 2.3. Under the assumptions of Theorem 2.2, we have for $1 \leq k \leq n$,

$$
\lambda_{n}^{(k)} \leq \frac{k}{x_{n-k+1}^{r}} .
$$

Moreover, we have $\lambda_{n}^{(n)} \geq \frac{1}{x_{1}^{r}}$.

Proof. First of all, we establish the fact that

$$
\mathbf{y}^{*}\left(\frac{1}{\left[x_{i}, x_{j}\right]^{r}}\right) \mathbf{y} \leq \frac{k \mathbf{y}^{*} \mathbf{y}}{x_{n-k+1}^{r}}
$$

for $\mathbf{y} \in V^{k}$, a $k$-dimensional subspace of the $n$-dimensional complex vector space $\mathbb{C}^{n}$ consisting of vectors that have zero entries in the coordinates at $1, \ldots, n-k$, namely, $\mathbf{y}=\left(y_{1}, \ldots, y_{n}\right)^{T}$ with $y_{i}=0$ for $i=1, \ldots n-k$, where $\mathbf{y}^{*}$ denotes the complex conjugate transpose of $\mathbf{y}$. We also use $\bar{a}$ to represent the complex conjugate of a complex number $a$. We illustrate our claim by induction. It is clear that

$$
\mathbf{y}^{*}\left(\frac{1}{\left[x_{i}, x_{j}\right]^{r}}\right) \mathbf{y}=\frac{\bar{y}_{n} y_{n}}{x_{n}^{r}}=\frac{\mathbf{y}^{*} \mathbf{y}}{x_{n}^{r}}
$$

where $\mathbf{y} \in V^{1}$. Thus it is true for $k=1$. Assume for the purpose of induction that the claim is true for some $1 \leq k<n$. We show the case for $k+1$.

Denote by $C_{e}$ the $e$-th column of the matrix $\left(\frac{1}{\left[x_{i}, x_{j}\right]^{r}}\right)$. Let $\mathbf{y} \in V^{k+1}$. Observe that

$$
\mathbf{y}^{*}\left(\frac{1}{\left[x_{i}, x_{j}\right]^{r}}\right) \mathbf{y}=\mathbf{y}^{*} C_{n-k} y_{n-k}+\mathbf{y}^{*} C_{n-k+1} y_{n-k+1}+\cdots+\mathbf{y}^{*} C_{n} y_{n} .
$$

Let $\mathbf{z} \in V^{k}$ such that $z_{i}=y_{i}$ if $i \neq n-k$ and $z_{i}=0$ if $i=n-k$. Therefore there is a copy of $\mathbf{z}^{*}\left(\frac{1}{\left[x_{i}, x_{j}\right]^{*}}\right) \mathbf{z}$ embedded into the previous expression. In fact,

$$
\begin{aligned}
\mathbf{y}^{*}\left(\frac{1}{\left[x_{i}, x_{j}\right]^{r}}\right) \mathbf{y}= & \mathbf{y}^{*} C_{n-k} y_{n-k}+\bar{y}_{n-k} \frac{1}{\left[x_{n-k}, x_{n-k+1}\right]^{r}} y_{n-k+1}+\cdots+ \\
& +\bar{y}_{n-k} \frac{1}{\left[x_{n-k}, x_{n}\right]^{r}} y_{n}+\mathbf{z}^{*}\left(\frac{1}{\left[x_{i}, x_{j}\right]^{r}}\right) \mathbf{z} .
\end{aligned}
$$

A routine computation shows

$$
\begin{aligned}
\left|\mathbf{y}^{*} C_{n-k} y_{n-k}\right| & \leq \frac{1}{x_{n-k}^{r}}\left(\left|\bar{y}_{n-k}\right|+\cdots+\left|\bar{y}_{n}\right|\right)\left|y_{n-k}\right| \\
& \leq \frac{1}{x_{n-k}^{r}}\left(\left|y_{n-k}\right|^{2}+\frac{1}{2} \sum_{i=1}^{k}\left(\left|y_{n-k}\right|^{2}+\left|y_{n-k+i}\right|^{2}\right)\right) \\
& =\frac{1}{2 x_{n-k}^{r}}\left((k+1)\left|y_{n-k}\right|^{2}+\mathbf{y}^{*} \mathbf{y}\right)
\end{aligned}
$$

since $|a b| \leq \frac{1}{2}\left(|a|^{2}+|b|^{2}\right)$ for any complex numbers $a$ and $b$. Similarly, we have

$$
\left|\bar{y}_{n-k} \frac{1}{\left[x_{n-k}, x_{n-k+1}\right]^{r}} y_{n-k+1}+\cdots+\bar{y}_{n-k} \frac{1}{\left[x_{n-k}, x_{n}\right]^{r}} y_{n}\right| \leq \frac{1}{2 x_{n-k}^{r}}\left((k-1)\left|y_{n-k}\right|^{2}+\mathbf{y}^{*} \mathbf{y}\right) .
$$


Furthermore by the induction hypothesis we have also

$$
\mathbf{z}^{*}\left(\frac{1}{\left[x_{i}, x_{j}\right]^{r}}\right) \mathbf{z} \leq \frac{k \mathbf{z}^{*} \mathbf{z}}{x_{n-k+1}^{r}} \leq \frac{k \mathbf{z}^{*} \mathbf{z}}{x_{n-k}^{r}}=\frac{k\left(\mathbf{y}^{*} \mathbf{y}-\left|y_{n-k}\right|^{2}\right)}{x_{n-k}^{r}} .
$$

Putting (9)-(11) together, by (8) we have

$$
\mathbf{y}^{*}\left(\frac{1}{\left[x_{i}, x_{j}\right]^{r}}\right) \mathbf{y} \leq \frac{(k+1) \mathbf{y}^{*} \mathbf{y}}{x_{n-k}^{r}} .
$$

This completes the induction and the claim is proved.

Let $\mathbf{v}_{1}, \ldots, \mathbf{v}_{n-k}$ be $n-k$ orthonormal vectors that span $\left(V^{k}\right)^{\perp}$. Then by the above claim (7) we get that

$$
\begin{aligned}
& \frac{k}{x_{n-k+1}^{r}} \geq \max _{0 \neq \mathbf{y} \in V^{k}} \frac{\mathbf{y}^{*}\left(\frac{1}{\left[x_{i}, x_{j}\right]^{*}}\right) \mathbf{y}}{\mathbf{y}^{*} \mathbf{y}}=\max _{0 \neq \mathbf{y} \perp \mathbf{v}_{1}, \ldots, \mathbf{v}_{n-k}} \frac{\mathbf{y}^{*}\left(\frac{1}{\left[x_{i}, x_{j}\right]^{*}}\right) \mathbf{y}}{\mathbf{y}^{*} \mathbf{y}}
\end{aligned}
$$

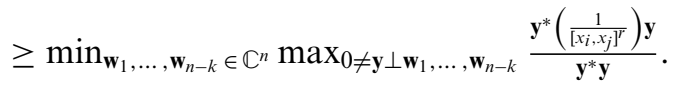

Now we apply the Courant-Fisher theorem (see [27]) to obtain

$$
\lambda_{n}^{(k)} \leq \frac{k}{x_{n-k+1}^{r}}
$$

as desired. Thus the first part of Theorem 2.3 is proved.

We proceed to show the lower bound of $\lambda_{n}^{(n)}$. We let $U^{1}$ be the 1-dimensional subspace of $\mathbb{C}^{n}$ consisting of vectors that have zero entries in the coordinates at $2, \ldots, n$. Again, by the Courant-Fisher theorem, we immediately have

$$
\begin{aligned}
\lambda_{n}^{(n)} & =\max _{\mathbf{w}_{1}, \ldots, \mathbf{w}_{n-1} \in \mathbb{C}^{n}} \min _{0 \neq \mathbf{y} \perp \mathbf{w}_{1}, \ldots, \mathbf{w}_{n-1}} \frac{\mathbf{y}^{*}\left(\frac{1}{\left[x_{i}, x_{j}\right]^{*}}\right) \mathbf{y}}{\mathbf{y}^{*} \mathbf{y}} \\
& \geq \min _{0 \neq \mathbf{y} \perp \mathbf{u}_{1}, \ldots, \mathbf{u}_{n-1}} \frac{\mathbf{y}^{*}\left(\frac{1}{\left[x_{i}, x_{j}\right]^{*}}\right) \mathbf{y}}{\mathbf{y}^{*} \mathbf{y}}=\min _{0 \neq \mathbf{y} \in U^{1}} \frac{\mathbf{y}^{*}\left(\frac{1}{\left[x_{i}, x_{j}\right]^{T}}\right) \mathbf{y}}{\mathbf{y}^{*} \mathbf{y}}=\frac{1}{x_{1}^{r}},
\end{aligned}
$$

where $\mathbf{u}_{1}, \ldots, \mathbf{u}_{n-1}$ denotes $n-1$ orthonormal vectors spanning $\left(U^{1}\right)^{\perp}$ and $\mathbf{y}=$ $\left(y_{1}, 0, \ldots, 0\right)^{T} \in U^{1}$. Therefore, $\lambda_{n}^{(n)} \geq \frac{1}{x_{1}^{r}}$. This completes the proof.

By Theorem 2.3, the second assertion of part (ii) of Theorem 2.2 also follows immediately. We also remark that $\frac{n-q}{x_{q+1}^{r}} \geq \lambda_{n}^{(n-q)}$ for $0 \leq q<n$ as a consequence of this analysis.

3. On the largest and mediate eigenvalues of reciprocal power LCM matrices. In this section we first turn our attentions to the asymptotic behavior of the largest eigenvalue of the reciprocal power LCM matrices. Later we consider the asymptotic behavior of some mediate eigenvalues. We begin with the following results.

THEOREM 3.1. Let $r>0$ be a real number, $q \geq 0$ a given arbitrary integer and $\left\{x_{i}\right\}_{i=1}^{\infty}$ an arbitrary given strictly increasing infinite sequence of positive integers. Let $\lambda_{n}^{(1)} \leq \ldots \leq \lambda_{n}^{(n)}$ be the eigenvalues of the $n \times n$ reciprocal power LCM matrix $\left(\frac{1}{\left[x_{i}, x_{j}\right]^{]}}\right)$defined on the set $S_{n}:=\left\{x_{1}, \ldots, x_{n}\right\}$. Then each of the following is true: 
(i) If $\sum_{i=1}^{\infty} \frac{1}{x_{i}^{r}}<\infty$, the sequence $\left\{\lambda_{n}^{(n-q)}\right\}_{n=q+1}^{\infty}$ converges. Furthermore, we have

$$
\lim _{n \rightarrow \infty} \lambda_{n}^{(n-q)} \leq \sum_{i=1}^{\infty} \frac{1}{x_{i}^{r}}
$$

(ii) In particular, the sequence $\left\{\lambda_{n}^{(n-q)}\right\}_{n=q+1}^{\infty}$ converges if $r>1$. Furthermore, we have

$$
\lim _{n \rightarrow \infty} \lambda_{n}^{(n-q)} \leq \zeta(r)
$$

Proof. First the Cauchy's interlacing theorem tells us that for any integer $n \geq q+1$, we have

$$
\lambda_{n}^{(n-q)} \leq \lambda_{n+1}^{(n+1-q)}
$$

Second, since $\lambda_{n}^{(i)}>0$ for $1 \leq i \leq n$ and $\sum_{i=1}^{n} \lambda_{n}^{(i)}=\sum_{i=1}^{n} \frac{1}{x_{i}^{r}}$, we then deduce

$$
\lambda_{n}^{(n-q)}<\sum_{i=1}^{n} \frac{1}{x_{i}^{r}}<\sum_{i=1}^{\infty} \frac{1}{x_{i}^{r}} .
$$

Suppose now that $\sum_{i=1}^{\infty} \frac{1}{x_{i}^{r}}<\infty$. Then there exists a constant $C>0$ such that $\sum_{i=1}^{\infty} \frac{1}{x_{i}^{r}}<C$. Thus we have

$$
\lambda_{n}^{(n-q)}<C
$$

Therefore the sequence $\left\{\lambda_{n}^{(n-q)}\right\}_{n=q+1}^{\infty}$ is monotonic increasing and has an upper bound, and so it is convergent. From (12) the upper bound of the limit in the second statement of part (i) follows immediately.

To show part (ii), let $r>1$. Then the value of the Riemann zeta function $\zeta(r)<\infty$. But

$$
\sum_{i=1}^{\infty} \frac{1}{x_{i}^{r}} \leq \sum_{i=1}^{\infty} \frac{1}{i^{r}}=\zeta(r) .
$$

So we have $\sum_{i=1}^{\infty} \frac{1}{x_{i}^{r}}<\infty$. Now the first statement of part (ii) follows immediately from the first statement of part (i). For the second statement of part (ii), it just follows from (13) and the second statement of part (i). The proof of Theorem 3.1 is complete.

COROLlARY 3.1. Let $r>0$ be a real number, $q \geq 0$ a given arbitrary integer and $\left\{x_{i}\right\}_{i=1}^{\infty}$ an arbitrary given strictly increasing infinite divisor chain sequence of positive integers, namely $x_{i} \mid x_{j}$ whenever $1 \leq i \leq j$. Let $\lambda_{n}^{(1)} \leq \ldots \leq \lambda_{n}^{(n)}$ be the eigenvalues of the $n \times n$ reciprocal power LCM matrix $\left(\frac{1}{\left[x_{i}, x_{j}\right]^{]}}\right)$defined on the set $S_{n}:=\left\{x_{1}, \ldots, x_{n}\right\}$. Then the sequence $\left\{\lambda_{n}^{(n-q)}\right\}_{n=q+1}^{\infty}$ converges and we have

$$
\lim _{n \rightarrow \infty} \lambda_{n}^{(n-q)} \leq\left(\frac{2}{x_{1}}\right)^{r} \cdot \frac{1}{2^{r}-1}
$$


Proof. Since $x_{1}|\ldots| x_{n} \mid \ldots$, we get $x_{i} \geq x_{1} \cdot 2^{i-1}$ for all $i \geq 1$. So we deduce that

$$
\sum_{i=1}^{\infty} \frac{1}{x_{i}^{r}} \leq \sum_{i=1}^{\infty} \frac{1}{x_{1}^{r} \cdot 2^{r(i-1)}}=\frac{1}{x_{1}^{r}} \cdot \sum_{i=0}^{\infty} \frac{1}{2^{r i}}=\left(\frac{2}{x_{1}}\right)^{r} \cdot \frac{1}{2^{r}-1} .
$$

Then Corollary 3.1 follows immediately from Theorem 3.1.

COROLlaRY 3.2. Let $r \geq 1$ be a real number, $q \geq 0$ a given arbitrary integer. Let $\lambda_{n}^{(1)} \leq \ldots \leq \lambda_{n}^{(n)}$ be the eigenvalues of the $n \times n$ reciprocal power LCM matrix $\left(\frac{1}{[i ! j,]^{r}}\right)=$ $\left(\frac{1}{((\max (i, j)) !)^{r}}\right)$ defined on the set $S_{n}:=\{1 !, 2 !, \ldots, n !\}$. Then the sequence $\left\{\lambda_{n}^{(n-q)}\right\}_{n=q+1}^{\infty}$ converges and we have

$$
\lim _{n \rightarrow \infty} \lambda_{n}^{(n-q)} \leq e-1
$$

Proof. Since $x_{i}=i$ ! for all $i \geq 1$ and $r \geq 1$, we have

$$
\sum_{i=1}^{\infty} \frac{1}{x_{i}^{r}}=\sum_{i=1}^{\infty} \frac{1}{(i !)^{r}} \leq \sum_{i=1}^{\infty} \frac{1}{i !}=e-1
$$

Thus Corollary 3.2 follows immediately from Theorem 3.1.

COROLLARY 3.3. Let $r>0$ be a real number, $q \geq 0$ and $a \geq 1$ given arbitrary integers. Let $\lambda_{n}^{(1)} \leq \ldots \leq \lambda_{n}^{(n)}$ be the eigenvalues of the $n \times n$ reciprocal power LCM matrix $\left(\frac{1}{\left[a^{i-1}, a^{j-1}\right]^{r}}\right)=\left(\frac{1}{a^{r \cdot(\max [i, j]-1)}}\right)$ defined on the set $S_{n}:=\left\{1, a, \ldots, a^{n-1}\right\}$. Then the sequence $\left\{\lambda_{n}^{(n-q)}\right\}_{n=q+1}^{\infty}$ converges and we have

$$
\lim _{n \rightarrow \infty} \lambda_{n}^{(n-q)} \leq \frac{a^{r}}{a^{r}-1}
$$

Proof. Since $x_{i}=a^{i-1}$ for all $i \geq 1$, we derive that

$$
\sum_{i=1}^{\infty} \frac{1}{x_{i}^{r}}=\sum_{i=0}^{\infty} \frac{1}{a^{r i}}=\frac{a^{r}}{a^{r}-1}
$$

Then Corollary 3.3 follows immediately from Theorem 3.1.

EXAMPLE 3.1. Let $r>0$ be a real number, $q \geq 0$ a given arbitrary integer. Let $\lambda_{n}^{(1)} \leq \ldots \leq \lambda_{n}^{(n)}$ be the eigenvalues of the $n \times n$ reciprocal power LCM matrix $\left(\frac{1}{\left[2^{i-1}, 2^{j-1}\right]^{r}}\right)=\left(\frac{1}{2^{r \cdot(\max [i, j\}-1)}}\right)$ defined on the set $S_{n}:=\left\{1,2, \ldots, 2^{n-1}\right\}$. Then by Corollary 3.3 we know that the sequence $\left\{\lambda_{n}^{(n-q)}\right\}_{n=q+1}^{\infty}$ converges and

$$
\lim _{n \rightarrow \infty} \lambda_{n}^{(n-q)} \leq \frac{2^{r}}{2^{r}-1}
$$

By Theorem 2.3 we have $\lim _{n \rightarrow \infty} \lambda_{n}^{(n)} \geq 1$. For $r=1$, by using Maple 9.5 we find that

$$
\lim _{n \rightarrow \infty} \lambda_{n}^{(n)}=1.454035950283418071052764297816596195350 \ldots
$$


REMARK. Although by Theorem 3.1 we know that $\lim _{n \rightarrow \infty} \lambda_{n}^{(n-q)}$ exists for any given integer $q \geq 0$ if $\sum_{i=1}^{\infty} \frac{1}{x_{i}^{r}}<\infty$, the problem of computing the exact limit remains wide open. In the forthcoming works, we will explore this interesting topic.

Finally we consider the asymptotic behavior of mediate eigenvalues of the reciprocal power LCM matrices. We have the following results.

THEOREM 3.2. Let $r>1$ be a real number and $n$ be a positive integer. Suppose $\left\{x_{i}\right\}_{i=1}^{\infty}$ is an arbitrary strictly increasing infinite sequence of positive integers. Let $\lambda_{n}^{(1)} \leq \ldots \leq \lambda_{n}^{(n)}$ be the eigenvalues of the $n \times n$ reciprocal power LCM matrix $\left(\frac{1}{\left[x_{i}, x_{j}\right]^{]}}\right)$defined on the set $S_{n}:=\left\{x_{1}, \ldots, x_{n}\right\}$. Then each of the following is true:

(i) If $q$ is an integer satisfying $C n \leq q \leq n-1$, where $0<C<1$ is a constant, then we have

$$
\lambda_{n}^{(n-q)} \leq \frac{c}{n^{r-1}}
$$

where $c>0$ is a constant.

(ii) For any integer-valued function $k(n)$ such that $1 \leq k(n) \leq C n$, where $0<C<1$ is a constant, the sequence $\left\{\lambda_{n}^{(k(n))}\right\}_{n}$ converges. Further we have $\lim _{n \rightarrow \infty} \lambda_{n}^{(k(n))}=0$. In particular, we have $\lim _{n \rightarrow \infty} \lambda_{l n}^{(t n-e)}=0$ for any given integers $e$, $t$ and $l$ such that $e \geq 0$ and $1 \leq t \leq l-1$.

Proof. Observe that $x_{k} \geq k$. Then from Theorem 2.3 we deduce that

$$
\lambda_{n}^{(n-q)} \leq \frac{n-q}{(q+1)^{r}} .
$$

But the condition $C n \leq q \leq n-1$, where $0<C<1$ is a constant, implies that we can find a constant $c$ so that

$$
\frac{n-q}{(q+1)^{r}} \leq \frac{c}{n^{r-1}}
$$

In fact, we can take $c=\frac{1-C}{C^{r}}>0$ since $0<C<1$. So part (i) follows immediately from (14) and (15).

On the other hand, by Theorem 2.2 (i) we know that $\lambda_{n}^{(n-q)}>0$. Since $r>1$, by part (i) and taking the limit we then obtain that $\lim _{n \rightarrow \infty} \lambda_{n}^{(n-q)}=0$. Clearly from $1 \leq k(n) \leq C n$ we can deduce that $(1-C) n \leq n-k(n) \leq n-1$. Hence picking $q=n-$ $k(n)$ one gets $\lim _{n \rightarrow \infty} \lambda_{n}^{(k(n))}=0$ as required. We then conclude part (ii). This completes the proof of Theorem 3.2.

THEOREM 3.3. Let $n$ be a positive integer. Suppose $\left\{x_{i}\right\}_{i=1}^{\infty}$ is an arbitrary given strictly increasing infinite sequence consisting of prime numbers except for finite terms. Let $\lambda_{n}^{(1)} \leq \ldots \leq \lambda_{n}^{(n)}$ be the eigenvalues of the reciprocal LCM matrix $\left(\frac{1}{\left[x_{i}, x_{j}\right]}\right)$ defined on the set $S_{n}:=\left\{x_{1}, \ldots, x_{n}\right\}$. Then each of the following is true:

(i) If $q$ is an integer satisfying $C n \leq q \leq n-1$, where $0<C<1$ is a constant, then we have

$$
\lambda_{n}^{(n-q)} \leq \frac{c}{\log (q+1)}
$$

where $c>0$ is a constant.

(ii) The same statements as that of Theorem 3.2 (ii). 
Proof. By Cauchy interlacing theorem it suffices to show the results for the case that $\left\{x_{i}\right\}_{i=1}^{\infty}$ consists of all primes except for finite terms. W.L.O.G. we may let $x_{i}=p_{i}$ denote the $i$-th prime. Following a well-known fact that $\frac{1}{6} i \log i<p_{i}$ (see Theorem 4.7 of [3]), by Theorem 2.3 we have

$$
\lambda_{n}^{(n-q)} \leq \frac{n-q}{p_{q+1}}<\frac{n-q}{\frac{1}{6}(q+1) \log (q+1)} .
$$

Since $C n \leq q \leq n-1$ with $0<C<1$ a constant, there exists a constant $c$ so that

$$
\frac{n-q}{\frac{1}{6}(q+1) \log (q+1)} \leq \frac{c}{\log (q+1)} .
$$

Actually, one may take $c=\frac{6(1-C)}{C}$. Then (16) as well as (17) concludes part (i). Note that $\lambda_{n}^{(n-q)}>0$ and $q \geq C n$ implying $q \rightarrow \infty$ when $n \rightarrow \infty$. Therefore, taking the limit in part (i), we get $\lim _{n \rightarrow \infty} \lambda_{n}^{(n-q)}=0$. As in the proof of Theorem 3.2, letting $q=n-k(n)$ part (ii) then follows immediately. So Theorem 3.3 is proved.

\section{REFERENCES}

1. E. Altinisik, B. E. Sagan and N. Tuglu, GCD matrices, posets, and nonintersecting paths, Linear Multilinear Algebra 53 (2005), 75-84.

2. T. M. Apostol, Arithmetical properties of generalized Ramanujan sums, Pacific J. Math. 41 (1972), 281-293.

3. T. M. Apostol, Introduction to analytic number theory (Springer-Verlag, 1976).

4. S. Beslin and S. Ligh, Greatest common divisor matrices, Linear Algebra Appl. 118 (1989), 69-76.

5. K. Bourque and S. Ligh, Matrices associated with arithmetical functions, Linear Multilinear Algebra 34 (1993), 261-267.

6. K. Bourque and S. Ligh, Matrices associated with classes of arithmetical functions, J. Number Theory 45 (1993), 367-376.

7. K. Bourque and S. Ligh, Matrices associated with classes of multiplicative functions, Linear Algebra Appl. 216 (1995), 267-275.

8. W. Cao, On Hong's conjecture for power LCM matrices, Czechoslovak Math. J. 57 (2007), 253-268.

9. L. E. Dickson, History of the theory of numbers, Vol. I (Chelsea Publ., 1999).

10. G. H. Hardy and E. M. Wright, An introduction to the theory of numbers, Fourth Edition (Oxford University Press, 1960).

11. P. Haukkanen and I. Korkee, Notes on the divisibility of LCM and GCD matrices, International J. Math. and Math. Science 6 (2005), 925-935.

12. P. Haukkanen, J. Wang and J. Sillanpää, On Smith's determiant, Linear Algebra Appl. 258 (1997), 251-269.

13. H. Hedenmalm, P. Lindqvist and K. Seip, A Hilbert space of Dirichlet series and systems of dilated functions in $L^{2}(0,1)$, Duke Math. J. 86 (1997), 1-37.

14. T. Hilberdink, Determinants of multiplicative Toeplitz matrices, Acta Arith 125 (2006), $265-284$.

15. S. Hong, Bounds for determinants of matrices associated with classes of arithmetical functions, Linear Algebra Appl. 281 (1998), 311-322.

16. S. Hong, On the Bourque-Ligh conjecture of least common multiple matrices, J. Algebra 218 (1999), 216-228.

17. S. Hong, Gcd-closed sets and determinants of matrices associated with arithmetical functions, Acta Arith. 101 (2002), 321-332. 
18. S. Hong, On the factorization of LCM matrices on gcd-closed sets, Linear Algebra Appl. 345 (2002), 225-233.

19. S. Hong, Factorization of matrices associated with classes of arithmetical functions, Colloq. Math. 98 (2003), 113-123.

20. S. Hong, Notes on power LCM matrices, Acta Arith. 111 (2004), 165-177.

21. S. Hong, Nonsingularity of matrices associated with classes of arithmetical functions, J. Algebra 281 (2004), 1-14.

22. S. Hong, Nonsingularity of least common multiple matrices on gcd-closed sets, J. Number Theory 113 (2005), 1-9.

23. S. Hong, Nonsingularity of matrices associated with classes of arithmetical functions on lcm-closed sets, Linear Algebra Appl. 416 (2006), 124-134.

24. S. Hong and R. Loewy, Asymptotic behavior of eigenvalues of greatest common divisor matrices, Glasgow Math. J. 46 (2004), 551-569.

25. S. Hong and R. Loewy, Asymptotic behavior of the smallest eigenvalue of matrices associated with completely even functions $(\bmod r)$, submitted.

26. S. Hong, K. P. Shum and Q. Sun, On nonsingular power LCM matrices, Algebra Colloq. 13 (2006), 689-704.

27. R. Horn and C. R. Johnson, Matrix analysis (Cambridge University Press, 1985).

28. S. Hwang, Cauchy's interlace theorem for eigenvalues of Hermitian matrices, Amer. Math. Monthly 111 (2004), 157-159.

29. I. Korkee and P. Haukkanen, On meet and join matrices associated with incidence functions, Linear Algebra Appl. 372 (2003), 127-153.

30. M. Li, Notes on Hong's conjectures of real number power LCM matrices, J. Algebra 315 (2007), 654-664.

31. P. Lindqvist and K. Seip, Note on some greatest common divisor matrices, Acta Arith. 84 (1998), 149-154.

32. P. J. McCarthy, A generalization of Smith's determinant, Canad. Math. Bull. 29 (1986), $109-113$.

33. I. Niven and H. Zuckerman, An introduction to the theory of numbers, Third Edition (Wiley, New York, 1960). 2004).

34. J. Sándor and B. Crstici, Handbook of number theory II (Kluwer Academic Publishers,

35. H. J. S. Smith, On the value of a certain arithmetical determinant, Proc. London Math. Soc. 7 (1875-1876), 208-212. $564-578$

36. A. Wintner, Diophantine approximations and Hilbert's space, Amer. J. Math. 66 (1944),

37. J. Zhao, S. Hong, Q. Liao and K. P. Shum, On the divisibility of power LCM matrices by power GCD matrices, Czechoslovak Math. J. 57 (2007), 115-125. 